\title{
INTENTO SUICIDA: VEINTICINCO AÑOS DE INVESTIGACIÓN EN ESTUDIANTES MEXICANOS DE EDUCACIÓN MEDIA SUPERIOR
}

\author{
Martha Córdova Osnaya, José Carlos Rosales Pérez y Aldebarán Rosales Córdova \\ UNAM, Universidad Anahuac \\ México
}

\begin{abstract}
RESUMEN
De acuerdo con la Organización Mundial de la Salud (OMS) el intento suicida representa un desafío para la salud pública. Este trabajo tiene como objetivo describir la información que se reporta en las investigaciones que se han efectuado en estudiantes mexicanos de educación media superior, en quienes se registró el intento suicida mediante la revisión de las investigaciones publicadas en revistas científicas entre 1993 y 2017. Se identificaron 17 artículos con tres formas diferentes de preguntas, además de la no especificación de la manera en que se registró el intento suicida. El $76.48 \%$ de los artículos no detalló el año en que se hizo la recolección de sus datos. La edad de los participantes fluctuó de entre 12 a 24 años. El $88.23 \%$ de las investigaciones utilizaron el porcentaje como el indicador de la presencia de intento suicida, el cual osciló de 7.20 a $12.50 \%$. Cuatro de las 17 investigaciones ( $23.52 \%$ ) reportaron: 1 ) el porcentaje de la presencia de intento suicida por género (en los varones fluctuó de 3.10 a $7.70 \%, y$ en las mujeres de 9.70 a $15.60 \%$ ), y 2 ) la razón intento suicida mujer/hombre cuyo valor fue de 2 a 3.
\end{abstract}

Palabras Clave:

intento suicida, jóvenes, estudiantes de preparatoria, comportamiento suicida, México.

\section{SUICIDAL ATTEMPT: TWENTY-FIVE YEARS OF RESEARCH IN MEXICAN STUDENTS OF UPPER SECONDARY EDUCATION}

\begin{abstract}
According to the World Health Organization, the suicide attempt represents a challenge for public health. The objective of this work is to describe the information that is reported in the investigations that have been conducted on baccalaureate Mexican students in whom the suicide attempt was recorded in articles published in scientific journals between the years of 1993 and 2017. Were identified 17 articles with three different forms of questions in addition to the non-specification of how he recorded the suicide attempt. $76.48 \%$ of the articles did'nt specify the year in which the data collection was carried out. The age of the participants ranged from 12 to 24 years. $88.23 \%$ of the researches used the percentage as the indicator of the presence of suicide attempt which ranged from $7.20 \%$ to $12.50 \%$. Four of the 17 investigations ( $23.52 \%)$ reported: 1 ) the percentage of the presence of suicidal attempt by gender: in men it ranged from $3.10 \%$ to $7.70 \%$, and in women from $9.70 \%$ to $15.60 \%$; 2 ) the suicide attempt ratio woman / man whose value was from 2 to 3.
\end{abstract}

Keywords:

suicidal attempt, young, students of baccalaureate, suicidal behavior, México.

BitÁcora del ARtículo:

| Recibido: 12 de Diciembre de 2018 | Aceptado: 29 de Marzo de 2019 | Publicado en línea: Julio - Diciembre de 2019 | 
Revisión de la literatura | Intento suicida: Veinticinco años de investigación... I Córdova-Osnaya, Rosales-Pérez eq Rosales-Córdova

\title{
Autoría y Derechos de Propiedad Intelectual
}

\section{INTENTO SUICIDA: VEINTICINCO AÑOS DE INVESTIGACIÓN EN ESTUDIANTES MEXICANOS DE EDUCACIÓN MEDIA SUPERIOR}

\author{
Martha Córdova Osnaya, José Carlos Rosales Pérez y Aldebarán Rosales Córdova \\ UNAM, Universidad Anahuac \\ México
}

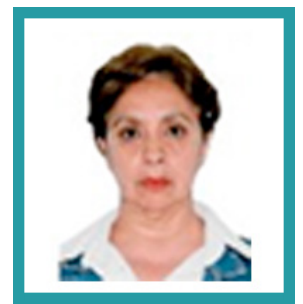

Martha Córdova Osnaya

FES Iztacala, UNAM

Correo: m.cordova@unam.mx

Licenciada en Psicología y maestría en Farmacología Conductual por la Universidad Nacional Autónoma de México (UNAM). Doctorado en Antropología por la Escuela Nacional de Antropología e Historia (ENAH). Miembro del Sistema Nacional de Investigadores (Conacyt) desde hace 15 años. Profesora titular " $C$ " en el área de Métodos Cuantitativos en la carrera de Psicología en Facultad de Estudios Profesionales Iztacala-UNAM.

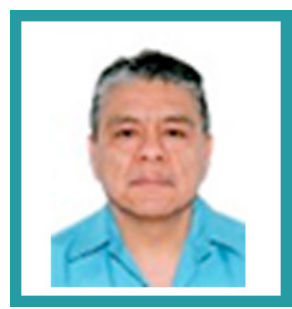

José Carlos Rosales Pérez FES Iztacala, UNAM Correo: jcrosales@unam.mx

Licenciado en Psicología, maestría en Enseñanza Superior, y doctorado en Psicología de la Salud por la Universidad Nacional Autónoma de México. Profesor titular " $C$ " en la asignatura de Procesos Estadísticos en la carrera de Psicología de la FES Iztacala-UNAM. Miembro del Sistema Nacional de Investigadores por el Consejo Nacional de Ciencia y Tecnología (Conacyt). Participación como autor o coautor en 30 artículos de investigación y 13 capítulos de libros.

\section{CONTRIBUCIÓN DE LOS Autores}

Los tres autores en forma conjunta desarrollamos cada etapa del artículo desde su planeación hasta la última corrección.

\section{AgRADECIMIENTOS}

\begin{abstract}
Agradecemos a la Universidad Nacional Autónoma de México campus Iztacala (Facultad de Estudios Superiores Iztacala) y a la Universidad Anáhuac México, por el apoyo recibido para la elaboración del presente estudio a través del tiempo otorgado a los autores para la realización del trabajo de investigación y de escritura.
\end{abstract}

\section{Datos de Filiación de los Autores}

Martha Córdova Osnaya, FES Iztcala UNAM | José Carlos Rosales Pérez, FES Iztcala UNAM | Aldebarán Rosales Córdova, Universidad Anahuac

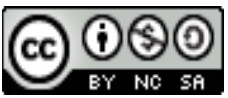

Copyright: ( 2019 Córdova-Osnaya, M.; Rosales-Pérez, J. C.; \& Rosales-Córdova, A.

Este es un artículo de acceso abierto distribuido bajo los términos de la licencia Creative Commons Reconocimiento-NoComercial 4.0 Internacional, por lo que su contenido gráfico y escrito se puede compartir, copiar y redistribuir total o parcialmente sin necesidad de permiso expreso de sus autoras con la única condición de que no se puede usar con fines directamente comerciales y los términos legales de cualquier trabajo derivado deben ser los mismos que se expresan en la presente declaración. La única condición es que se cite la fuente con referencia a la Revista Digital Internacional de Psicología y Ciencia Social y a sus autoras. 


\section{TABLA DE CONTENIDO}

INTRODUCCIÓN

RESULTADOS

Formas de registrar el intento suicida, fuente y opciones de respuesta, 34

Discusión

Forma de registrar el intento suicida, 37

Fuente de las preguntas, 37

Opciones de respuesta de las preguntas, 37

Formato y aplicación de las preguntas, 37

Palabras usadas en las preguntas, 38

Temporalidad del registro de intento suicida por medio de las preguntas, 38

Año en que se hizo la captura o recolección de los datos, 39

Entidad federativa a la cual pertenecen los participantes, 39

Tamaño de la muestra y tipo de muestreo, 39

Edad de los participantes, 39

Îndice de participantes por género, 40

Presencia de intento suicida mediante frecuencia o porcentaje (en muestra total y por género), 40

CONCLUSIONES

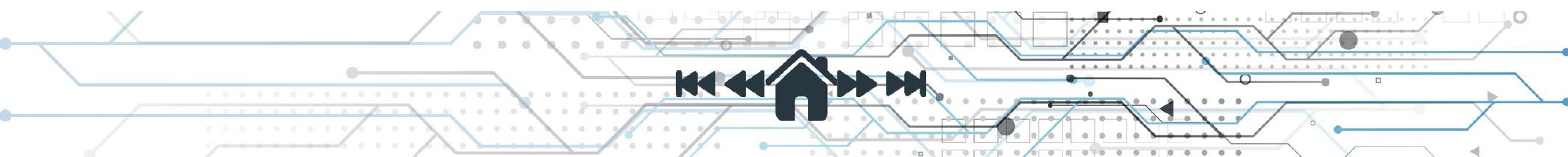




\section{INTRODUCCIÓN}

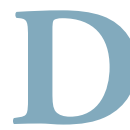

e acuerdo con la Organización Mundial de la Salud (OMS), tanto el suicidio como el intento suicida son importantes desafíos para la salud pública (OMS, 2018). De acuerdo con dicha institución el impacto de un suicidio en el aspecto psicológico, familiar y social es inmenso, por lo que a partir de 1999 lanzó una iniciativa mundial para la prevención del suicidio por medio de un programa conocido como Supre (Suicide Prevention), invirtiendo recursos en investigación, difusión y programas específicos para "profesionales de la salud, educadores, agencias sociales, gobiernos, legisladores, comunicadores sociales, funcionarios judiciales, familias y comunidades" (OMS, 2006:, p. v).

Respecto al intento suicida la OMS indica que: 1) la frecuencia del intento suicida es más alta que el suicidio: "Hay indicios de que, por cada adulto que se suicidó, posiblemente más de otros 20 intentaron suicidarse" (OMS, 2014: sección Mensajes clave, párr. 1), "Por cada suicidio, hay muchas más tentativas de suicidio cada año" (OMS, 2018: sección Datos y cifras, párr. 2); 2) incremento del comportamiento suicida en todo el mundo: "En los últimos 45 años las tasas de suicidio han aumentado en un 60\% a nivel mundial" (OMS, 2012: sección El problema, párr.2); 3) el factor de riesgo significativo para el suicidio es el intento suicida: "Entre la población en general, un intento de suicidio no consumado es el factor individual de riesgo más importante" (OMS, 2018: sección Datos y cifras, párr. 2); 4) experiencias estrechamente relacionadas con conductas suicidas, como conflictos, desastres, violencia, abusos, pérdidas y sensación de aislamiento (OMS, 2018); 5) cambia en los grupos de edades de acuerdo con el género: las mujeres adultas intentan suicidarse de 2 a 3 veces más que los hombres adultos (OMS, 2009); 6) la investigación del comportamiento suicida es complejo por los múltiples elementos involucrados, en donde interaccionan factores biológicos, psicológicos, sociales, ambientales y culturales (OMS, 2014); 7) limitada fidedignidad y calidad de los datos: "En todo el mundo es insuficiente la disponibilidad y calidad de los datos sobre el suicidio y los intentos de suicidio" (OMS, 2018: sección Calidad de los datos, párr. 1), de modo que desde 2012 la OMS indica que "La fiabilidad de los sistemas de certificación y notificación de los suicidios requiere importantes mejoras" (OMS, 2012: sección Desafíos y obstáculos, párr. 2).
El objetivo primordial de la OMS es desarrollar estrategias de prevención tanto del suicidio como del intento suicida, por lo que señala:

Las diferencias transnacionales en los patrones de suicidio y los cambios en las tasas, características y métodos de suicidio ponen de relieve la necesidad de que cada país mejore la integridad, calidad y oportunidad de sus datos concernientes al suicidio. Esto incluye el registro civil de suicidios, los registros hospitalarios de intentos de suicidio, y los estudios representativos a escala nacional que recopilen información sobre intentos de suicidio autonotificados (OMS, 2018: sección Calidad de los datos, párr. 2).

Respecto a los intentos de suicidio autonotificados en México, en 1995 el Instituto Nacional de Estadística, Geografía e Informática (INEGI) inició la publicación detallada de intentos de suicidio y suicidios con cobertura temporal de 1990-1994 por medio de Cuadernos denominados "Estadísticas de Intentos de Suicidio y Suicidios" (INEGI, 1995); los datos reportados en dichos Cuadernos son derivados de las agencias del Ministerio Público. Se publicaron 12 números de esos cuadernos; el último número se publicó en 2007 con cobertura temporal de 2005 (INEGI, 2007). A partir de 2007, el INEGI dejó de publicar la información respecto al intento suicida por medio de estos Cuadernos, para dar paso sólo a la publicación de suicidios, los cuales se captan mediante los registros de las estadísticas de mortalidad, sobre todo de las accidentales y violentas en las agencias del Ministerio Público (INEGI, 2017).

Hoy México no tiene una institución oficial que agrupe los registros de las estadísticas de los intentos del suicidio ocurridos en todo el país. La información de dicho fenómeno reposa en las publicaciones científicas; al respecto, Borges, Orozco y Medina-Mora (2012), con datos de la Encuesta Nacional de Adicciones elaborada en 2008 en mexicanos cuyas edades oscilaron de 12 a 65 años, reportaron un porcentaje de intento suicida en muestra total (hombres y mujeres) de $8.99 \%$ (7.87\% en hombres y $9.59 \%$ en mujeres).

La presente investigación responderá algunas preguntas referentes al intento suicida en estudiantes mexicanos de preparatoria derivada de las investigaciones en artículos científicos, tales como ¿de qué formas se ha registrado?, ¿cuál es la forma más frecuente de reportar el intento suicida?, ¿cuál es el porcentaje de intento suicida?, ¿cuáles son los estados de la República Mexicana en donde se ha registrado el intento suicida?, ¿se ha incrementado el índice de intento suicida con el transcurrir del tiempo?, ¿con qué frecuencia se ha considerado el género en el reporte de intento suicida? y ¿cuál es la razón intento suicida mujer/hombre? 
Así, el objetivo del presente estudio es describir la información que se reporta en las investigaciones que se han desarrollado en estudiantes mexicanos de educación media superior, en quienes se registró el intento suicida mediante la revisión de las investigaciones publicadas en revistas científicas entre 1993 y 2017.

\section{MÉtodo}

Estudio observacional, descriptivo, retrospectivo, y transversal (Méndez, Namihira, Moreno y Sosa, 2012).

En la búsqueda de los artículos científicos se utilizaron dos descriptores de búsqueda tanto en español como en inglés: Intento Suicida (Suicide Attempt) y autolesión (Self-Arm) en las siguientes bases de datos: Medline (http:// www.ncbi.nlm.nih.gov/pubmed/), Dirección General de Bibliotecas de la Universidad Nacional Autónoma de México (dgb.unam.mx), Salud Pública de México (http:// saludpublica.mx/index.php/spm/search), Centro de Documentación Científica de Psiquiatría y Salud (http://inprf-cd. gob.mx/), Red de Revistas Científicas de América Latina y el Caribe, España y Portugal (http://www.redalyc.org), y Google Académico (http://scholar.google.com.mx).

La indagación de artículos científicos se hizo durante los meses de agosto-diciembre de 2017. Los criterios de inclusión fueron: 1) que el artículo fuera sido publicado entre 1993 y 2017 ; 2) que en la sección de participantes éstos fueran estudiantes mexicanos de educación media superior; 3) haber registrado de algún modo el intento suicida en estudiantes mexicanos de preparatoria, y 4) reportar el número de estudiantes o porcentaje que presentó intento suicida en estudiantes mexicanos de educación media superior.

Considerando los criterios de inclusión, la búsqueda en las bases de datos arrojó la localización de 17 resúmenes de artículos publicados en el transcurso de 25 años.

\section{Procedimiento}

El análisis de los 17 artículos completos consistió en identificar y describir los siguientes rubros: formas de registrar el intento suicida; año en que se hizo la captura o recolección de datos; estado de la República Mexicana al cual pertenecen los participantes; tamaño de la muestra y tipo de muestreo; edad de los participantes; porcentaje de participantes por género; presencia de intento suicida mediante frecuencia o por índice tanto en muestra total como por género, y el reporte de la razón intento suicida mujer/hombre.

\section{Resultados}

En los 17 artículos se identificaron cuatro escenarios respecto a la forma de registrar el intento suicida; tres preguntas diferentes y la ausencia de especificación de cómo se registró el intento suicida.

La información recabada de los 17 artículos (año en que se hizo la captura o recolección de datos, tamaño de la muestra y tipo de muestreo, edad de los participantes, índice de participantes por género, presencia de intento suicida y reporte de la razón intento suicida mujer/hombre) fue vertida en tres tablas; en cada una se agrupó dicha información de acuerdo con el estado de la República Mexicana a la que pertenecen los estudiantes; en los casos en donde hubo más de un artículo y la misma entidad federativa, la información fue organizada de modo descendente de acuerdo con el año de publicación del artículo.

En la tabla 1 se describe la información de los artículos que utilizaron la forma de registrar el intento suicida por medio de la pregunta: “¿Alguna vez, a propósito, te has herido, intoxicado o hecho daño con el fin de quitarte la vida?".

En la tabla 2 (ver en anexo) se detalla la información de dos artículos; cada uno de ellos usó una pregunta diferente para el registro del intento suicida; dichas preguntas fueron: "En los últimos cinco años, ¿has intentado alguna vez quitarte la vida?" y "En los últimos 12 meses, ¿las veces que ha intentado suicidarse en realidad?".

En la tabla 3 (ver en anexo) se describen las investigaciones que no especificaron la manera de registrar el intento suicida (pero sí detallaron su porcentaje).

\section{Formas de registrar el intento suicida, fuente y opciones de respuesta}

\section{Pregunta. "¿Alguna vez, a propósito, te has herido, intoxicado o hecho daño con el fin de quitarte la vida?"}

Nueve de los 17 artículos (52.94\%; tabla 1) integraron esta pregunta en un cuestionario autoaplicado con más reactivos. Los artículos señalaron como fuente de dicha pregunta a González-Forteza, Mariño, Rojas, Mondragón y Medina-Mora (1998). Las opciones de respuesta utilizadas por los autores fueron de dos tipos: No/Una vez/Más de una vez —opciones de respuesta derivada de la fuente original (Cantoral-Cancino, y Betancur-Ocampo, 2011; Chávez, Pérez, Macías y Páramo, 2004; González-Forteza, Álvarez-Ruiz, Saldaña-Hernández, Carreño-García, Chávez y Pérez-Hernández, 2005; Monge, Cubillas, Román y Valdez, 2007; Rivera, y Andrade,

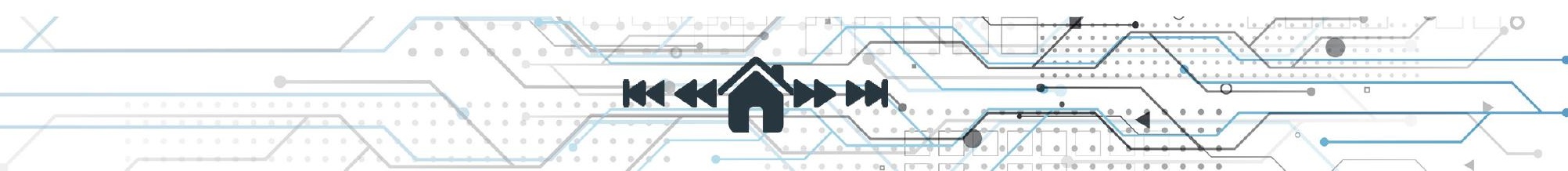


2006)_, y Sí/No (González-Fuentes, y Andrade, 2013; Palacios, Sánchez y Andrade, 2010; Pérez-Amezcua, Rivera-Rivera, Atienzo, De Castro, Leyva-López y Chávez-Ayala, 2010).

\section{Pregunta. "En los últimos cinco años, ¿has intentado alguna vez quitarte la vida?"}

Sólo hay un artículo - tabla 2 (Espinoza-Gómez, Zepeda-Pamplona, Bautista-Hernández, Hernández-Suárez, Newton-Sánchez y Plasencia-García, 2010)_ cuyos autores usaron en las opciones de respuesta Sí/No, reactivo integrado en una encuesta que se aplicó a todos los alumnos de primer ingreso mediante el examen médico automatizado (EMA), diseñado y validado por la Universidad Nacional Autónoma de México.

\section{Pregunta. "En los últimos 12 meses, ¿las veces que ha intentado suicidarse en realidad?"}

Sólo hay un artículo — tabla 2 (Hidalgo-Rasmussen e Hidalgo-San Martín, 2015)_ en el que los autores tradujeron la pregunta de la encuesta de comportamiento de riesgo juvenil del Centro Nacional para la Prevención de Enfermedades Crónicas y Promoción de la Salud que indica la American Psychological Association; en dicha encuesta las opciones de respuesta son: 0 veces/ 1 vez/ 2-3 veces/ 4-5 veces/ 6 o más veces. Los autores codificaron las respuestas de los estudiantes como Sí/No. La respuesta "Sí" fue considerada de 1 vez hasta 6 o más veces. La respuesta "No"; cero veces. Pregunta que los autores integraron en un cuestionario en línea.

No especificación de la forma en que se efectuó el registro de intento suicida

Seis artículos (35.29\%) no especificaron la forma de registrar el intento suicida, pero sí señalaron el uso de una pregunta integrada en un cuestionario autoaplicado en la sección de datos generales, puntualizando además el índice encontrado de intento suicida (Valadez-Figueroa, Amezcua-Fernández y Amezcua-Casas, 2015; Valadez-Figueroa, Amezcua-Fernández, González-Gallegos y Alfaro-Alfaro, 2009; Valadez-Figueroa, Amezcua-Fernández, Quintanilla-Montolya y González-Gallegos, 2005; Valadez-Figueroa, Chávez-Hernández, Vargas-Valdez y Ochoa-Orendain, 2016; Valadez-Figueroa, Quintanilla-Montolya, González-Gallegos y Amezcua-Fernández, 2005; Valadez, Amezcua, González, Montes y Vargas, 2011).

\section{Año en que se hizo la captura o recolección de datos}

Sólo cuatro de los 17 artículos reportaron el año en que se hizo la captura de los datos (23.52\%). Tres de estas cuatro investigaciones utilizaron, para registrar el intento suicida, la pregunta "¿Alguna vez, a propósito, te has herido, intoxicado o hecho daño con el fin de quitarte la vida?" (tabla 1); de estas tres investigaciones, dos de sus participantes pertenecen al estado de Guanajuato, reportando el año de captura de los datos en 2002-2003 (Chávez et al., 2004; González-Forteza et al., 2005), y la tercera investigación a las 32 entidades federativas de la República Mexicana cuya recopilación de los datos se efectuó en 2007 (Pérez-Amezcua et al., 2010). La cuarta investigación usó para el registro del intento suicida la pregunta "En los últimos cinco años, ¿has intentado alguna vez quitarte la vida?" (tabla 2), aplicada a estudiantes de preparatoria de Colima en 2006 (Espinoza-Gómez et al., 2010).

Entidad federativa a la cual pertenecen los participantes de las investigaciones

Los estados de la República Mexicana que han sido objeto de investigación de intento suicida por sus estudiantes de educación media superior se ha hecho tanto en una investigación transversal de todos los estados de la República Mexicana, como es el caso de Pérez-Amezcua et al. (2010), así como investigaciones que sólo refieren a estudiantes pertenecientes a un solo estado (tablas 1-3). En este último caso están Chipas, Colima, Sonora, Guanajuato, Ciudad de México y Jalisco.

\section{Tamaño de la muestra y tipo de muestreo}

El 64.70\% (11 de las 17) de las investigaciones utilizaron un muestreo aleatorio con tamaños de muestras que oscilaron de 343 a 12,424 estudiantes. Las muestras no aleatorias fueron $29.41 \%$ (cinco artículos) con tamaños de muestras de 263 a 1,000 estudiantes. Hay un caso (5.8\%) donde se usó toda la población de estudiantes de primer ingreso integrado por 5,484 estudiantes (tablas 1-3).

\section{Edad de los participantes}

Tres de las 17 investigaciones no reportaron la edad de los participantes (Rivera y Andrade, 2006 (tabla 1); Valadez-Figueroa et al., 2015; Valadez-Figueroa et al., 2016 (tabla 3). Una investigación indicó la edad de los participantes sólo mediante el promedio, el cual fue de 15.75 años con una DE = 1 (González-Forteza et al., 2005 (tabla 1).

Los restantes 13 artículos especificaron la edad de los participantes por medio del rango de edad, el cual osciló de 12 a 24 años (tablas 1-3). Dos de esas 13 investigaciones además de especificar el rango de edad 
de los participantes también indicaron el promedio y la DE; las medias reportadas fueron 16.7 y 16.5 con DE de 1.36 y 1 respectivamente (González-Fuentes y Andrade, 2013; Cantoral-Cancino y Betancur, 2011).

\section{Índice de participantes por género}

El índice de mujeres en la muestra de los 17 artículos osciló de 50 a $69.58 \%$, y de los hombres de 30.54 a 50\% (tablas 1-3).

\section{Presencia de intento suicida mediante frecuencia o porcentaje en muestra total, y por género}

De los nueve artículos que reportaron la presencia de intento suicida en muestra total por medio de la pregunta "¿Alguna vez, a propósito, te has herido, intoxicado o hecho daño con el fin de quitarte la vida?", dos de estas investigaciones especificaron la presencia mediante la frecuencia (Rivera y Andrade, 2006; González-Fuentes y Andrade, 2013) y siete artículos detallaron la presencia de intento suicida por medio del índice (Chávez et al., 2004; González-Forteza et al., 2005; Palacios y Andrade, 2007; Palacios et al., 2010; Monge et al., 2007; Pérez-Amezcua et al., 2007; Cantoral-Cancino y Betancurt-Ocampo, 2011). El índice en muestra total de las siete investigaciones que lo enunciaron osciló de 7.20 a $12.50 \%$ (González-Forteza et al., 2005; Palacios et al., 2010, respectivamente). Referente a la presencia de intento suicida por género, sólo dos artículos lo describieron con el índice: en Guanajuato, cuyo índice en hombres fue de $3.10 \%$ y en mujeres de $10.70 \%$ (González-Forteza et al., 2005), y en Sonora, en donde el índice en hombres fue de $5.80 \%$ y en mujeres de $15.60 \%$ (Monge et al., 2007).

La publicación que especifica el registro de intento suicida con la pregunta "En los últimos cinco años, ¿has intentado alguna vez quitarte la vida?" aplicada a estudiantes de Colima (tabla 2), detalló un índice en muestra total de $7.30 \%$ (sin su frecuencia). Respecto al género, sólo se pormenorizó por frecuencia; 111 hombres y 289 mujeres (Espinosa-Gómez et al., 2010).

La investigación que utilizó para el registro de intento suicida la pregunta "En los últimos 12 meses, ¿las veces que ha intentado suicidarse en realidad?" (tabla 2), aplicada a estudiantes de Jalisco reportó índices (sin frecuencia) en muestra total de $7.60 \%$, en hombres de $5.20 \%$ y en mujeres de $9.70 \%$ (Hidalgo-Rasmussen y Hidalgo-San Martín, 2015).

Los seis artículos que no especificaron la manera de registrar el intento suicida cuyos participantes fueron estudiantes de la Ciudad de Guadalajara reportaron en muestra total tanto la frecuencia como el índice (tabla 3); en estas seis investigaciones se reportaron dos porcentajes: 9.66\% (Valadez-Figueroa et al., 2009; Valadez-Figueroa et al., 2011; Valadez-Figueroa et al., 2015; Valadez-Figueroa et al., 2016) y $11.95 \%$ (Valadez-Figueroa, Amezcua et al., 2005; Valadez-Figueroa, Quintanilla et al., 2005). Respecto al género, sólo una investigación de las seis especificó el reporte de intento suicida: hombres $7.70 \%$ y mujeres $11.90 \%$ (Valadez-Figueroa et al., 2009).

\section{Reporte de la razón intento suicida mujer/ hombre}

Cuatro de las 17 investigaciones especificaron la razón intento suicida mujer/hombre.

Dos de los cuatro artículos reportaron una razón de 3 (tres mujeres por cada hombre intentan suicidarse) con una diferencia estadísticamente significativa mujer/ hombre con una ji cuadrada (con $p=000$ y $p<0.001$ ). Ambos artículos utilizaron para el registro de intento suicida la pregunta "¿Alguna vez, a propósito, te has herido, intoxicado o hecho daño con el fin de quitarte la vida?" (tabla 1); los estudiantes de preparatoria participantes pertenecen al estado de Guanajuato (González-Forteza et al., 2005) y a Sonora (Monge et al., 2007).

El tercer artículo que señaló la razón intento suicida mujer/hombre, indica que sus estudiantes participantes pertenecen a la región centro sur de Jalisco. Se utilizó la pregunta "En los últimos 12 meses, ¿las veces que ha intentado suicidarse en realidad?" para el registro del intento suicida (tabla 2), la razón intento suicida mujer/ hombre tuvo un valor de 2 con el cálculo de razón de momios con significancia estadística de $\mathrm{p}<0.001(\mathrm{Hi}-$ dalgo-Rasmussen e Hidalgo-San Martín, 2015).

El cuarto y último artículo en el que también se reportó la razón intento suicida mujer/hombre con un valor de 1.5 sin referencia estadística, los estudiantes de preparatoria pertenecen a la Ciudad de Guadalajara (tabla 3); en el artículo no se especificó la forma de registrar el intento suicida (Valadez-Figeroa et al., 2009).

\section{DisCUSIÓN}

De acuerdo con el objetivo de la presente investigación y los resultados encontrados, esta sección discurrirá en ocho aspectos del intento suicida en estudiantes mexicanos de educación media superior: forma de registrar el intento suicida; año en que se hizo la captura o recolección de los datos; estado de la República Mexicana al que pertenecen los participantes de las investigaciones; tamaño de la muestra y tipo de muestreo; edad de los participantes; índice de participantes por género; presencia de intento

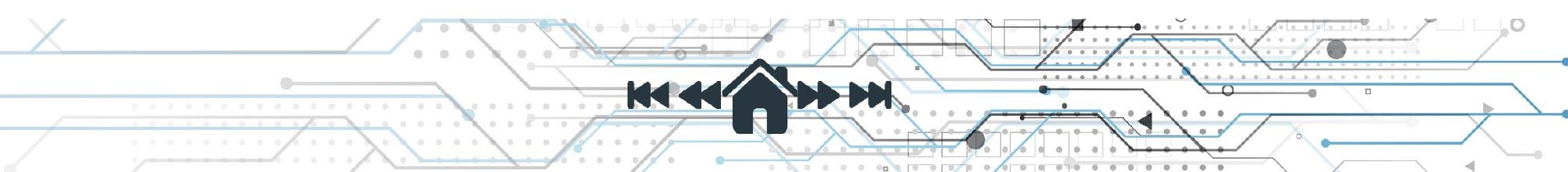


suicida mediante frecuencia o por índice; y reporte de la razón intento suicida mujer/hombre.

\section{Forma de registrar el intento suicida}

En estudiantes mexicanos de educación media superior los artículos científicos reportaron tres tipos diferentes de preguntas para el registro del intento suicida; además de estos tres tipos diferentes de registrar el intento suicida se debe añadir la carencia de especificación de la manera como se hizo el registro de intento suicida. Lo anterior conduce a los siguientes cuestionamientos: ¿cuál pregunta usar para hacer una investigación cuyo propósito sea registrar el intento suicida?, ¿se podría usar cualquiera de las tres preguntas y se obtendrían los mismos resultados?; la respuesta a las anteriores interrogantes trascienden el propósito del presente estudio; sin embargo se pondrán en la mesa de discusión algunos aspectos importantes a considerar para poder responder dichas preguntas de acuerdo con lo que informan las investigaciones respecto al intento suicida en estudiantes mexicanos de preparatoria.

\section{Fuente de las preguntas}

El que se presenten tres preguntas diferentes y la no especificación en el registro del intento suicida muestra diferentes fuentes: Instituto Mexicano de Psiquiatría para el caso de la pregunta " $i$ Alguna vez, a propósito, te has herido, intoxicado o hecho daño con el fin de quitarte la vida?" (usada en 52.94\% de los artículos); la American Psychological Association (2014) para la pregunta "En los últimos 12 meses, ¿las veces que ha intentado suicidarse en realidad?"; la Universidad Nacional Autónoma de México respecto a la pregunta "En los últimos cinco años, ¿has intentado alguna vez quitarte la vida?"; y la omisión de fuente en $35.29 \%$ de los artículos en donde no se especificó la manera de registrar el intento suicida.

La variabilidad anterior en el registro del intento suicida y su respectiva fuente en estudiantes mexicanos de educación media superior muestra la ausencia de acuerdo entre investigadores; al respecto se podría considerar lo siguiente: los reactivos o preguntas, así como instrumentos de medición, se derivan del marco teórico, de modo que la heterogeneidad del registro de intento suicida es un reflejo de la heterogeneidad de la concepción teórica de dicho concepto; también es relevante indicar que al efectuarse el registro de intento suicida, siendo éste un concepto teórico (y no una variable sociodemográfica) por medio de sólo un reactivo o pregunta, conlleva a obtener datos poco fidedignos; por otra parte, no especificar la manera de registrar el intento suicida de $35.29 \%$ de los artículos muestra una deficiencia metodológica que evidencia la poca importancia que se ha dado al registro del intento suicida.

\section{Opciones de respuesta de las preguntas}

Las tres preguntas reportadas utilizaron opciones de respuesta cerrada; tampoco hay información de cuál fue la opción de respuesta en $35.29 \%$ de las investigaciones que no especificaron la manera de registrar el intento suicida.

En el caso de las investigaciones que utilizaron las preguntas "¿Alguna vez, a propósito, te has herido, intoxicado o hecho daño con el fin de quitarte la vida?" y "En los últimos 12 meses, ¿las veces que ha intentado suicidarse en realidad?" cambiaron las opciones de respuesta politómica de la fuente original a dicotómica Sí/ No. De manera que las tres preguntas utilizaron la opción de respuesta cerrada Sí/No; dicha opción de respuesta indica que la investigación de intento suicida en México se inclina en general a identificar la presencia o ausencia de dicho fenómeno.

\section{Formato y aplicación de las preguntas}

Las cuatro formas de registrar el intento suicida (incluido la no especificación de la manera de registrar el intento suicida) se hizo integrando la pregunta en un cuestionario con más preguntas (incluso en la sección de preguntas generales), es decir, se efectuó de modo simultáneo el registro del intento suicida con el registro o medición de otras variables. Lo anterior conlleva a suponer que se diluye el registro de intento suicida con otras variables, cuestión que no sucede con el registro o medición de la ideación suicida (registro que aunque se hace también con la medición de otras variables, tiene una sección específica en el cuestionario por medio de un instrumento), de manera que el investigador olvida indicar en su publicación la manera en que se efectuó el registro del intento suicida, como sucedió con 35.29\% de las investigaciones, aspecto que ocurrió también en la publicación de otra investigación en estudiantes mexicanos de educación media superior que no fue contemplada en este estudio porque no cubrió el criterio de inclusión de reportar el intento suicida por medio de frecuencia o índice (Cubillas, Román, Valdez y Galaviz, 2012).

Por otra parte, en dos de estas cuatro maneras de registrar el intento suicida su aplicación se hizo por medio de un cuestionario en línea o automatizado (Espinoza-Gómez et al., 2010; Hidalgo-Rasmussen e Hidalgo-San Martín, 2015), modo de aplicación que abre una línea de investigación. 


\section{Palabras usadas en las preguntas}

En este apartado se desarrollarán dos secciones referentes al tipo de palabras utilizadas en las preguntas que registraron el intento suicida.

\section{Palabra: "intentado"}

Dos de las tres preguntas contienen la palabra "intenta$\mathrm{do}^{\prime \prime}$; la pregunta que no tiene dicha palabra es la utilizada en $52.94 \%$ de los 17 artículos científicos (" ¿Alguna vez, a propósito, te has herido, intoxicado o hecho daño con el fin de quitarte la vida?"); los autores de esta pregunta aseveran que identificaron en los estudiantes una confusión semántica entre intento e intención, de ahí que textualmente afirman que dicha pregunta "no contuvo la palabra 'intento' para evitar que se confundiera con 'intención'. De este modo, quedó más claro el indicador de la conducta suicida y más cercana al concepto que se pretende evaluar y que en la literatura científica se denomina 'intento de suicidio o intento suicida' " (González-Forteza et al., 1998: 168).

Lo anterior hace referencia al significado semántico comparativo entre intento suicida (un acto) e intención suicida (un pensamiento o cognición). Partiendo del supuesto de que los estudiantes mexicanos de preparatoria confunden intento con intención, entonces la manifestación del intento suicida debería ser diferente en los que respondieron a las preguntas que contienen las palabras "intentado suicidarte" comparado con los que respondieron la pregunta que no tienen dichas palabras.

En esta orientación es que se aporta lo siguiente: en estudiantes de educación media superior del mismo estado de la República Mexicana (Jalisco) aunque en publicaciones de diferentes años se encontraron los siguientes índices de intento suicida en muestra total; en todo el estado de Jalisco al usar la pregunta "¿Alguna vez, a propósito, te has herido, intoxicado o hecho daño con el fin de quitarte la vida?", un índice de $10 \%$ (Pérez-Amezcua et al., 2010), en la ciudad de Guadalajara sin especificar la manera de registrar el intento suicida un índice que oscila de 9.95 a $11.95 \%$ (Valadez-Figueroa et al., 2015; Valadez-Figueroa et al., 2009; Valadez-Figueroa, Amezcua-Fernández et al., 2005; Valadez-Figueroa et al., 2016; Valadez-Figueroa, Quintanilla-Montolya et al., 2005; Valadez et al., 2011), y en la región sur de Jalisco con la pregunta "En los últimos 12 meses, ¿las veces que ha intentado suicidarse en realidad?" 7.6\% (Hidalgo-Rasmussen e Hidalgo-San Martín, 2015). Con los anteriores resultados, ¿se podría afirmar que hay una confusión semántica entre "intento" e "intención" suicida?, pregunta cuya respuesta tendrán que plantear las futuras investigaciones.

\section{Palabras: "con el fin de quitarte la vida"}

Las palabras "con el fin de quitarte la vida" hacen referencia a la intencionalidad; varios artículos que usaron la pregunta "¿Alguna vez, a propósito, te has herido, intoxicado o hecho daño con el fin de quitarte la vida?", también registraron el "deseo de muerte" mediante una pregunta con tres opciones de respuesta: "No querían morir", "No les importaba si vivían o morían", y "Si buscaban la muerte" (Chávez et al., 2004; González-Forteza et al., 2005; Palacios et al., 2010; Cantoral-Cancino y Betancourt-Ocampo, 2013). Después de contestar, los estudiantes que se habían herido, cortado, intoxicado, o hecho daño con el fin de quitarse la vida señalaron que "No querían morir" en un índice que oscila de 13.8 a $19.5 \%$ (en muestra total), de ahí que surja entonces la pregunta: "¿qué sucede semánticamente en la frase 'con el fin de quitarte la vida' en los estudiantes mexicanos de educación media superior?". ¿Este índice de ambigüedad en el estudiante (de lastimarse o autolesionarse, pero no con la intención de quitarse la vida) también se presenta en las preguntas que contienen las palabras "intención suicida"? Avanzado un poco en las posibles respuestas a las anteriores preguntas es que el DSM-5 (Manual Diagnóstico y Estadístico de los Trastornos Mentales 5ed., American Psychiatric Association, 2014) propone el concepto de "autolesión no suicida", en el que la autolesión descarta la intencionalidad suicida, por lo que queda un amplio camino por recorrer por parte de los investigadores del comportamiento suicida para identificar la mejor pregunta para registrar la autolesión con y sin intencionalidad suicida en estudiantes mexicanos de preparatoria.

Temporalidad del registro de intento suicida por medio de las preguntas

Dos de las preguntas incluyen temporalidad del intento suicida (dos de los 17 artículos; $11.78 \%$ ): un intento suicida recordado cinco años antes o 12 meses antes. El $52.94 \%$ de los artículos (nueve de los 17) que utilizaron la pregunta "¿Alguna vez, a propósito, te has herido, intoxicado o hecho daño con el fin de quitarte la vida?", no tiene temporalidad, de modo que el estudiante puede responder al recuerdo del intento suicida que haya tenido en cualquier momento de su vida, incluso cuando era niño(a). De 35.29\% de los artículos no se tiene información de si la pregunta tiene temporalidad o no.

La asignación de temporalidad al acto de intento suicida implica abrir una línea de investigación; al respecto, de nuevo el DSM-5 ha presentado la propuesta de considerar el intento suicida (con intencionalidad suicida — definido con el nombre de "Trastorno de Com- 
portamiento Suicida" - ) como "actual" si no han trascurrido más de 12 meses desde el último intento, y en "remisión" si han transcurrido de 12 a 24 meses desde el último intento.

También la temporalidad de 12 meses es considerada en investigaciones nacionales efectuadas con mexicanos de entre 12 a 65 años con la pregunta "En los últimos 12 meses, ¿ha intentado quitarse la vida?" (Borges et al., 2012). Los autores de este artículo proponemos que en futuras investigaciones el registro del intento suicida en estudiantes de educación media superior conlleve temporalidad, sobre todo para que después de su detección se proceda a su atención terapéutica.

\section{Año en que se hizo la captura o recolección de los datos}

La OMS (2012) ha indicado que el comportamiento suicida ha cambiado con el transcurrir del tiempo y que se ha incrementado en los últimos años en todo el mundo. Con la descripción de los resultados de la presente investigación, ¿sería posible identificar el incremento del intento suicida en las últimas décadas en estudiantes mexicanos de educación media superior? Los años de publicación de los 17 artículos oscila de 2004 a 2016, y sólo cuatro de ellos indicaron el año en que se hizo la captura de los datos.

Para poder efectuar una comparación del intento suicida conforme transcurre el tiempo, es imprescindible el año en que se hizo la captura de los datos (no así, el año en que se publicó el artículo). Lo anterior conduce a un gran vacío de lo que está sucediendo respecto al intento suicida con el transcurrir del tiempo en estudiantes mexicanos de educación media superior, porque de $76.48 \%$ de las investigaciones se desconoce el año al que corresponden sus datos. Por consiguiente, no es posible identificar la existencia de un incremento en el intento suicida en estudiantes mexicanos de preparatoria.

\section{Entidad federativa a la cual pertenecen los participantes}

La OMS (2018) ha señalado que el comportamiento suicida varía según la población; respecto al intento suicida en estudiantes de educación media superior, ¿se podría decir que los distintos estados de la República Mexicana son poblaciones diferentes? Dicha diferencia podría ser explicada por la temporalidad, la cual no es posible determinar debido a la carencia de la especificación del año de la recolección de los datos en $76.48 \%$ de los artículos revisados. Sin embargo, puede ser de gran ayuda la investigación de Pérez-Amezcua et al. (2010), cuyos autores hicieron el registro del intento suicida en estudiantes mexicanos de preparatoria en los 32 esta- dos de la República Mexicana en 2007 con la pregunta "¿Alguna vez, a propósito, te has herido, intoxicado o hecho daño con el fin de quitarte la vida?". Los autores reportaron una variabilidad en el índice de intento suicida que oscila de $5 \%$ que corresponde al estado de Tamaulipas a $15 \%$ que pertenece a Tabasco. Con la información anterior se puede plantear la hipótesis de que los diferentes estados de la República Mexicana tienen un comportamiento diferencial respecto al intento suicida en estudiantes mexicanos de educación media superior, aspecto que es necesario investigar en el futuro.

\section{Tamaño de la muestra y tipo de muestreo}

En términos metodológicos es preferible tener muestras aleatorias porque brindan la posibilidad de generalizar los resultados que se obtienen en las investigaciones a la población donde se extrajo la muestra. El $64.70 \%$ de las 17 investigaciones utilizaron este tipo de muestreo con tamaños de muestras que oscilaron de 343 a 12,424 estudiantes. Es loable un índice de $64.70 \%$ con muestras aleatorias, porque no siempre es posible efectuar este tipo de muestreo en estudiantes de educación media superior debido a que el procedimiento de cálculo y extracción depende sobre todo del apoyo de las autoridades educativas de las instituciones participantes.

Por otra parte, las muestras no aleatorias fueron $29.41 \%$ con tamaños de muestras de 263 a 1,000 estudiantes; si bien con estas muestras la generalización de los resultados se encuentra limitada, no dejan de ser importantes por cuanto describen lo que sucede en la muestra. También el apoyo de las autoridades de las instituciones educativas es relevante en este tipo de muestreo; sin embargo, el apoyo sólo se limita a su autorización para la aplicación del cuestionario.

\section{Edad de los participantes}

De acuerdo con la OMS (2018), es muy importante considerar los grupos de edad en el estudio del comportamiento suicida porque éste cambia dependiendo del rango de edad al que se esté refiriendo. El rango de edad de los participantes en 13 de las 17 investigaciones revisadas en este trabajo oscila de 12 años a 24 años, rango de edad amplio para el tipo de fenómeno que se estudia porque se ha reconocido teóricamente que el comportamiento suicida se incrementa en la adolescencia y disminuye en la edad adulta (Wagner y Zimmerman, 2008); 12 años es un adolescente y 24 años es un adulto.

Sin embargo, el rango de edad es un aspecto que en el registro de intento suicida en estudiantes mexicanos de educación media superior se ha puesto poca atención, aspecto que se puede evidenciar en tres de las 17 investiga- 
ciones que no reportaron de manera alguna (por rango o por promedio) la edad de los participantes. También hay investigaciones - además de las contempladas en el presente estudio- que, al reportar el intento suicida de sus participantes, fusionaron en un solo grupo - como una población homogénea- dos niveles educativos (rangos de edades que oscilan de 10 a 22 años), como es el caso de unir estudiantes mexicanos de secundaria y preparatoria (González-Forteza, Villatoro, Alcántar, Medina-Mora, Fleiz, Bermúdez y Amador, 2002; González-Forteza, Mariño, Mondragón y Medina-Mora, 2000; González-Forteza et al., 1998; Mayer, Morales, Victoria y Ulloa, 2016; Villatoro, Alcantar, Medina-Mora, Fleiz, González-Forteza, Amador y Bermúdez , 2003), o la integración de un solo grupo homogéneo de participantes entre estudiantes mexicanos de preparatoria y universitarios (con una edad promedio de 18.5 anos; Unikel, Gómez-Peresmitré, y González-Forteza, 2006).

Por tanto, se considera conveniente que en futuras investigaciones se reduzca el rango de edad de los participantes formando parte de los criterios de inclusión.

\section{Índice de participantes por género}

Al estar integrada la muestra total tanto por hombres como por mujeres y registrar un fenómeno como el intento suicida, donde las mujeres manifiestan una proporción mayor respecto a los hombres, como lo ha manifestado la OMS (2009), el investigador debe tener cuidado de la manera en que reporta el índice. En caso de que tenga un número mayor de mujeres en su muestra, el porcentaje de muestra total "punteará alto", por lo que se recomienda, en caso de que se tenga una diferencia significativa del índice de participantes por género, se haga el reporte de porcentaje de intento suicida por género y no por muestra total. De modo que es necesario tener mesura en la interpretación de la presencia de intento suicida en muestra total de la investigación que utilizó $69.58 \%$ de mujeres y $30.54 \%$ de hombres (Rivera y Andrade, 2006).

\section{Presencia de intento suicida mediante frecuencia o porcentaje (en muestra total y por género)}

\section{Presencia de intento suicida en muestra total}

La OMS (2009) señaló el índice de intento suicida en muestra total en 2009 describiendo que en distintos países la presencia de intento suicida osciló de entre 3 y $5 \%$. A partir de 2014 la OMS solo enfatiza al intento suicida necesariamente referido con el suicidio; por cada adulto que se suicida hay quizá más de otros 20 que intentaron suicidarse (OMS, 2014), y para 2018 la OMS reitera de nuevo el intento suicida tomando como base al suicidio "Por cada suicidio, hay muchas más tentativas de suicidio cada año" (OMS, 2018, sección Datos y cifras, párr. 2). A partir de 2009 la OMS dejó de publicar el índice mundial de intento suicida en muestra total debido a la falta de datos fidedignos, de manera que una de sus propuestas para la prevención del suicidio es que "cada país mejore la integridad, calidad y oportunidad de sus datos concernientes al suicidio" (OMS, 2018, sección Calidad de los datos, párr. 2), y una de las formas que propone dicha institución son "los estudios representativos a escala nacional que recopilen información sobre intentos de suicidio autonotificados" (OMS, 2018, sección Calidad de los datos, párr. 2).

El porcentaje en muestra total fue el indicador de presencia de intento suicida que con mayor frecuencia se utilizó en las 17 investigaciones revisadas en el presente trabajo (15 de los 17 artículos); las otras dos investigaciones reportaron la presencia de intento suicida por medio de la frecuencia. Respecto a esto último, pormenorizar sólo la frecuencia de un fenómeno como el intento suicida es un dato muy ambiguo porque carece del referente total; es mejor la opción del índice porque representa la proporción del total.

El índice de presencia de intento suicida en muestra total de las 15 investigaciones que lo reportaron fluctuó de 7.20 a $12.50 \%$. La variabilidad implica una diferencia de $5.3 \%$; se podrían considerar las siguientes hipótesis como respuesta a dicha variabilidad: el tipo de pregunta que se usó para el registro del intento suicida, la entidad federativa a la que pertenecen los participantes, la edad de los participantes y el año de recolección de datos, entre otras. Dichas hipótesis podrían considerarse en futuras investigaciones.

Respecto a investigaciones nacionales se tiene el reporte de intento suicida en muestra total de $8.99 \%$; esta investigación se efectuó en 2008 mediante una encuesta con mexicanos cuyas edades oscilaron entre 12 a 65 años por medio del registro del intento suicida con la pregunta "En los últimos 12 meses, ¿ha intentado quitarse la vida?" (Borges et al., 2012); este índice de $8.99 \%$ es muy similar al $9 \%$ nacional que especifica Pérez-Amezcua et al. (2010) en estudiantes mexicanos de educación media superior cuya recolección de datos se hizo en 2007 por medio de la pregunta "¿Alguna vez, a propósito, te has herido, intoxicado o hecho daño con el fin de quitarte la vida?". Con estos dos porcentajes tan parecidos para 2007-2008 se podría afirmar que no importa la forma de la pregunta para el registro del intento suicida ni su temporalidad, ni la edad de los participantes, así como tam- 
poco el nivel de estudios ni la entidad federativa, aspectos que las futuras investigaciones tendrán que responder.

Por otra parte, si se considera el índice en muestra total como un indicador de la presencia de intento suicida en estudiantes mexicanos de educación media superior, y se tiene en cuenta que según la OMS el intento suicida se ha incrementado en todo el mundo en los últimos años, es importante confirmar en futuras investigaciones el decremento del índice en muestra total de intento suicida que reportan las publicaciones de Guanajuato; decremento de $1.4 \%$ en la publicación de 2004 (8.37\% en Chávez et al., 2004) a 2005 (7.19\% en González-Forteza et al., 2005), y la Ciudad de Guadalajara; decremento de 2\% (de 11.95 a 9.95\%) en las publicaciones de 2005 (11.95\% en Valadez-Figueroa, Amezcua-Fernández et al., 2005; Valadez-Figueroa, Quintanilla-Montolya et al., 2005) a las de 2009, 2011, 2015 y 2016 (9.95\% en Valadez et al., 2011; Valadez-Figueroa et al., 2009; Valadez-Figueroa et al., 2015; Valadez-Figueroa et al., 2016).

También es importante indicar que el reporte de la presencia de intento suicida se ha fusionado con otra variable, como es el caso de una investigación que no fue posible integrar en el presente estudio porque el reporte de intento suicida en estudiantes de preparatoria de Sonora se mezcló con la sintomatología depresiva (Cubillas et al., 2012), de manera que no fue posible identificar el índice de intento suicida.

\section{Presencia de intento suicida por género}

Desde 2009 la OMS ha señalado que las mujeres adultas intentan suicidarse de 2 a 3 veces más que los varones adultos, lo cual indica una diferencia de género digna de considerar en la investigación del intento suicida; sin embargo, sólo cuatro de los 17 artículos detallaron índices para hombres y para mujeres.

En estos cuatro artículos la presencia de intento suicida en los varones osciló de 3.10 a $7.70 \%$, y en las mujeres de 9.70 a $15.60 \%$. Es relevante indicar la diferencia de porcentaje entre hombres y mujeres, en donde el índice en mujeres es mayor que en los hombres.

Además de la diferencia de porcentaje entre hombres y mujeres, también se nota una variabilidad significativa dentro del mismo género: $4.6 \%$ considerando sólo a los varones y $5.9 \%$ teniendo en cuenta sólo a las mujeres; la pregunta que surge es: ¿por qué esa variabilidad dentro de un mismo género?; de nuevo, como en el caso de la muestra total, serían varias las hipótesis que se podrían plantear, las cuales requieren investigación.

Por otra parte, respecto al índice nacional por género, Borges et al. (2012) refirieron un nivel de $7.87 \%$ en hombres y de $9.59 \%$ en mujeres. Comparando los resultados de Borges et al. (2012) y los de la presente investigación (infortunadamente no hay reporte de intento suicida a nivel nacional por género en estudiantes mexicanos de preparatoria); en el caso de los hombres, el índice reportado por Borges et al. (2012) coincide con el límite superior del rango de los resultados de la presente investigación (7.87 y $7.70 \%$ respectivamente); en el caso de las mujeres, lo informado Borges et al. (2012) coincide con el límite inferior del rango de los resultados de la presente investigación (9.59 y $9.70 \%$ respectivamente). Sin embargo en este caso particular, las comparaciones (fuera de ser sólo descriptivas) son improcedentes porque se desconoce el año de recolección de los datos cuyos participantes fueron estudiantes mexicanos de preparatoria.

Por otra parte, el bajo índice de reporte del intento suicida por género $(23.52 \% ; 4$ de 17$)$ sugiere la necesidad de que en futuras investigaciones además de reportarlo en muestra total también se especifique por género.

\section{Reporte de la razón intento suicida mujer/ hombre}

La razón intento suicida mujer/hombre es el indicador más destacado que usa la OMS para la comparación por género en grupos de edades pertenecientes a una población; las mujeres muestran intentos suicidas de dos a tres veces más que los hombres (OMS, 2009).

Cuatro de las 17 investigaciones que reportaron el índice de intento suicida por género también señalaron la razón intento suicida mujer/hombre de dos a tres veces por cada hombre (redondeando el valor de 1.5 de la publicación de Valadez-Figeroa et al., 2009).

De modo que la comparación de los datos obtenidos de la presente investigación y lo que reporta la OMS respecto a la razón intento suicida mujer/hombre coincide; es relevante destacar que el dato que señala la OMS refiere al año 2009, lo que parece indicar que la razón intento suicida mujer/hombre no ha cambiado. Sin embargo, es apropiado considerar dicha coincidencia a la luz de las limitantes que se presentaron al registrar los intentos suicidas.

\section{Conclusiones}

En el contexto del requerimiento que la OMS ha señalado desde 2012, referente a mejorar por parte de cada país la notificación de la calidad de los datos tanto del intento suicida como del suicidio para incrementar su fiabilidad, es que los resultados del presente estudio muestran tanto desafíos como recomendaciones a ser consideradas por 
las futuras investigaciones del comportamiento suicida en México, sobre todo para aquellas cuyo propósito sea registrar el intento suicida en estudiantes de educación media superior.

Uno de los desafíos que se afrontarán será la elección de la manera de registrar el intento suicida. Es tiempo de que los investigadores trasciendan de constreñir el registro de intento suicida a sólo una pregunta como si fuera una variable sociodemográfica o de identificación personal (como preguntar por el género), e incluirla en un cuestionario para que forme parte de las preguntas de aspectos generales. El intento suicida es un constructo teórico, y como tal requiere que del marco teórico de referencia se deriven su definición y su instrumento de registro, de modo que el instrumento de registro de intento suicida integre además de presencia/ausencia, la intensidad, la temporalidad, la forma, etcétera. Lo anterior permitiría una perspectiva del fenómeno a registrar mucho más amplia, además de que se podrían hacer cálculos de fiabilidad y validez (teniendo de esta manera datos con mayor veracidad).

Otro reto al que los investigadores tendrán que enfrentarse implica la concepción teórica de intento suicida: el concepto de "intencionalidad/no intencionalidad de quitarse la vida" cuando existe un autodaño físico. Aspecto clave que debe trabajarse de manera teórica y por consiguiente incorporarse en el registro de intento suicida.

Otro desafío para la futura investigación en intento suicida es la inclusión del informe de sus datos por género. Aspecto que afecta no sólo la expresión de la presencia/ausencia del intento suicida, sino también las variables que se asocian a dicho fenómeno. Si bien el presente estudio no reportó las variables que se asocian al intento suicida en estudiantes mexicanos de preparatoria, es relevante señalar que los artículos que consideraron variables asociadas al intento suicida el análisis estadístico utilizado se efectuó en muestra total y no por género.

Por otra parte, respecto a algunas recomendaciones para la investigación futura del intento suicida producto de la presente investigación son: 1) reportar en la publicación el año de recolección de los datos; 2) acotar la edad de los participantes formando parte del criterio de inclusión para efectuar la investigación; 3) reportar la razón intento suicida mujer/hombre, y 4) hacer investigaciones en los diferentes estados de la República Mexicana.

Es importante indicar que una limitación de este estudio es tal vez no haber considerado todos los artículos publicados en revistas científicas referentes al intento suicida en estudiantes mexicanos de educación media superior; sin embargo, es una muestra representativa.

\section{Referencias}

American Psychiatric Association (2014). Manual Diagnóstico y Estadístico de los Trastornos Mentales (DSM-5), 5a ed. Madrid: Editorial Médica Panamericana.

Borges, G., Orozco, R., \& Medina-Mora, M. E. (2012). Índice de riesgo para el intento suicida en México. Salud Pública de México, 56(6), 595-606. https://doi.org/10.1590/S003636342012000600008

Cantoral-Cancino, D. C., \& Betancourt-Ocampo, D. (2011). Intento de suicidio y ambiente familiar en adolescentes de Tuxtla Gutiérrez, Chiapas. Revista de Educación y Desarrollo, 19, 59-66.

Cubillas, M. J., Román, R., Valdez, E. A., \& Galaviz, A. L. (2012). Depresión y comportamiento suicida en estudiantes de educación media superior en Sonora. Salud Mental, 35, 45-50.

Chávez, A. M., Pérez, R., Macías, L. F., \& Páramo, D. (2004). Ideación e intento suicida en estudiantes de nivel medio superior de la Universidad de Guanajuato. Acta Universitaria, 14(3), 12-20.

Espinoza-Gómez, F., Zepeda-Pamplona, V., Bautista-Hernández, V., Hernández-Suárez, C. M., Newton-Sánchez, O. A., \& Plasencia-García, G. (2010). Violencia doméstica y riesgo de conducta suicida en universitarios adolescentes. Salud Pública de México, 52, 213-219. https://doi.org/10.1590/ S0036-36342010000300005

González-Forteza, C., Álvarez-Ruiz, M., Saldaña-Hernández, A., Carreño-García, S., Chávez, A. M., \& Pérez-Hernández, R. (2005). Prevalence of deliberate self-arm in the state of Guanajuato, México: 2003. Social Behavior and Personality, 33(8), 777-792. https://doi.org/10.2224/ sbp.2005.33.8.777

González-Forteza, C., Mariño, C., Mondragón, L., \& MedinaMora, M. E. (2000). Intento de suicidio y uso del tiempo libre en adolescentes mexicanos. Psicología Conductual, $8(1), 147-152$.

González-Forteza, C., Mariño, C., Rojas, E., Mondragón, L., \& Medina-Mora, M. E. (1998). Intento de suicidio en estudiantes de la Ciudad de Pachuca, Hidalgo, y su relación con el malestar depresivo y el uso de sustancias. Revista Mexicana de Psicología, 15(2), 165-167.

González-Forteza, C., Villatoro, J., Alcántar, I., Medina-Mora, M. E., Fleiz, C., Bermúdez, P., \& Amador, N. (2002). Prevalencia de intento suicida en estudiantes adolescentes de la ciudad de México:1997 y 2000. Salud Mental, 25(6), 1-12.

González-Fuentes, M. B., \& Andrade, P. (2013). Auto-aceptación como factor de riesgo para el intento de suicidio en adolescentes. Salud \& Sociedad, 4(1), 26-35. https://doi. org/10.22199/S07187475.2013.0001.00002

Hidalgo-Rasmussen, C., \& Hidalgo-San Martín, A. (2015). Comportamientos de riesgo de suicidio y calidad de vida, por género, en adolescentes mexicanos, estudiantes de preparatoria. Ciência \& Saúde Coletiva, 20(11), 3437-3445. https://doi.org/10.1590/1413-812320152011.18692014

Instituto Nacional de Estadística, Geografía e Informática (1995). Estadística de intentos de suicidio y suicidios. Cuaderno número 1. México: INEGI. 
Instituto Nacional de Estadística, Geografía e Informática (2007). Estadísticas de intentos de suicidio y suicidios de los Estados Unidos Mexicanos 2005. Cuaderno número 12. México: INEGI.

Instituto Nacional de Estadística y Geografía (2017). Defunciones por suicidio por entidad federativa de residencia habitual de la persona fallecida según sexo, 2010 a 2016. Recuperado el 3 de julio de 2018 de dehttp:// www.beta.inegi.org. $\mathrm{mx} / \mathrm{app} / \mathrm{tabulados/pxweb/inicio.}$ html?rxid=75ada3fe-1e52-41b3-bf27-4cda26e957a7\&db $=$ Mortalidad \&px=Mortalidad 7

Mayer, P. A., Morales, N., Victoria, G., \& Ulloa, R. E. (2016). Adolescentes con autolesiones e ideación suicida: un grupo con mayor comorbilidad y adversidad psicosocial. Salud Pública de México, 58(3), 335-336. https://doi. org/10.21149/spm.v58i3.7893

Méndez, I., Namihira, D., Moreno, L., \& Sosa, C. (2012). El protocolo de investigación. Lineamientos para su elaboración y análisis. México: Trillas.

Monge, J. A., Cubillas, M. J., Román, R., \& Valdez, E. A. (2007). Intentos de suicidio en adolescentes en educación media superior y su relación con la familia. Psicología y Salud, 17(1), 45-51.

Organización Mundial de la Salud (2006). Prevención del Suicidio. Recurso para consejeros. Trastornos mentales y cerebrales. Departamento de Salud Mental y Abuso de Sustancias. Recuperado el 5 de noviembre de 2018 de https://www.who.int/mental health/media/counsellors spanish.pdf?ua=1

Organización Mundial de la Salud (2009). Prevención del suicidio. Un instrumento para policías, bomberos y otros socorristas de primera línea. Departamento de Salud Mental y Abuso de Sustancias. Recuperado el 13 de junio de 2018 de https://www.who.int/mental health/ prevention/suicide/resource_responders_spanish. pdf?ua=1

Organización Mundial de la Salud (2012). Prevención del suicidio (SUPRE). Nota descriptiva con fecha de agosto de 2012. Recuperado el 30 de octubre de 2018 de https://www.who.int/mental_health/prevention/suicide/ suicideprevent/es/

Organización Mundial de la Salud (2014). Prevención del suicidio. Un imperativo global. Resumen ejecutivo. Recuperado el 13 de junio de 2018 de https://www.who. int/mental_health/suicide-prevention/exe_summary_ spanish.pdf?ua=1

Organización Mundial de la Salud (2018). Suicidio. Nota descriptiva con fecha de 31 de enero de 2018. Recuperado el 25 de junio de 2018 de http://www.who. int/mediacentre/factsheets/fs398/es/

Palacios, J. R., \& Andrade, P. (2007). Desempeño académico y conductas de riesgo en adolescentes. Revista de Educación y Desarrollo, 7, 5-16.

Palacios, J. R., Sánchez, B., \& Andrade, P. (2010). Intento de suicidio y búsqueda de sensaciones en adolescentes. Revista Intercontinental de Psicología y Educación, 12(1), 53-75.

Pérez-Amezcua, B., Rivera-Rivera, L., Atienzo, E., De Castro, F., Leyva-López, A., \& Chávez-Ayala, R. (2010). Prevalencia y factores asociados a la ideación e intento suicida en adolescentes de educación media superior de la República Mexicana. Salud Pública de México, 52(4), 324-333.

Rivera, M. E., \& Andrade, P. (2006). Recursos individuales y familiares que protegen al adolescente del intento suicida. Revista Intercontinental de Psicología y Educación, 8 (2), 23-40.

Unikel, C., Gómez-Peresmitré, G., \& González-Forteza, C. (2006). Suicidal Behaviour, risky eating behaviours and psychosocial correlates in Mexican female students. European Eating Disorders Review, 14, 414-421. https:// doi.org/10.1002/erv.707

Valadez-Figueroa, I., Amezcua-Fernández, R., \& Amezcua-Casas, M. (2015). La psicopatología posterior al intento suicida adolescente y sus mecanismos de defensa o adaptación. Acta Universitaria, 25(2), 16-23.

Valadez-Figueroa, I., Amezcua-Fernández, R., GonzálezGallegos, N., \& Alfaro-Alfaro, N. (2009). Psicopatología del adolescente con intento suicida y diferencias de género. Anales de Psiquiatría, 25(6), 265-274.

Valadez-Figueroa, I., Amezcua-Fernández, R., QuintanillaMontolya, R., \& González-Gallegos, N. (2005). Familia e intento suicida en el adolescente de Educación Media Superior. Archivos en Medicina Familiar, 7(3), 69-78.

Valadez-Figueroa, I., Chávez-Hernández, A. M., Vargas-Valdez, V., \& Ochoa-Orendain, M. C. (2016). Persistencia de los pensamientos de muerte posteriores a la tentativa suicida en una muestra de jóvenes mexicanos. Revista de Educación y Desarrollo, 39, 15-26.

Valadez-Figueroa, I., Quintanilla-Montolya, R., GonzálezGallegos, N., \& Amezcua-Fernández, R. (2005). El papel de la familia en el intento suicida del adolescente. Salud Pública de México, 47(1), 1-2. https://doi.org/10.1590/ S0036-36342005000100001

Valadez, I., Amezcua, R., González, N., Montes, R., \& Vargas, V. (2011). Maltrato entre iguales e intento suicida en sujetos adolescentes escolarizados. Revista Latinoamericana de Ciencias Sociales, Niñez y Juventud, 2(9), 783-796.

Villatoro, J. A., Alcantar, M. I., Medina-Mora, M. E., Fleiz, C. M., González-Forteza, C., Amador, N. G., \& Bermúdez P. (2003). El intento suicida y el consumo de drogas en adolescentes. ¿Dos problemas entrelazados? Revista SESAM, 2(1), 5-12.

Wagner, B. M., \& Zimmerman, J. H. (2008). Influencias del desarrollo en la suicidalidad entre adolescentes. Aspectos cognitivos, emocionales y de neurociencia. En Thomas E. Ellis (dir.), Cognición y suicidio. Teoría, investigación y terapia. México: Manual Moderno. 


\section{ANEXO}

Tabla 1.

Descripción de la información de los artículos que utilizaron la forma de registrar el intento suicida por medio de la pregunta: "¿Alguna vez, a propósito, te has herido, intoxicado o hecho daño con el fin de quitarte la vida?"

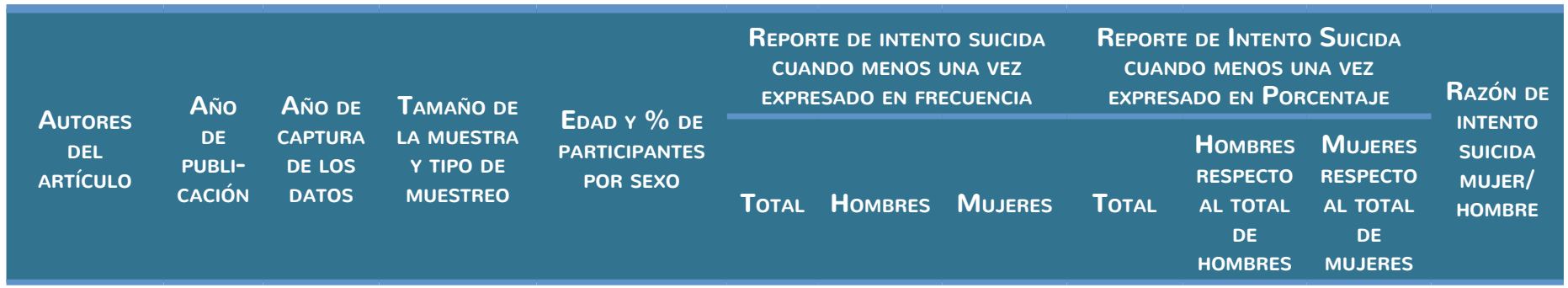

\begin{tabular}{|c|c|c|c|c|c|c|c|c|c|c|c|}
\hline $\begin{array}{l}\text { Chávez, } \\
\text { Pérez, } \\
\text { Macías, \& } \\
\text { Páramo. }\end{array}$ & 2004 & $\begin{array}{l}2002- \\
2003\end{array}$ & $\begin{array}{l}2,532 \\
\text { estudiantes } \\
\text { Muestra } \\
\text { aleatoria }\end{array}$ & $\begin{array}{c}\text { De } 13 \text { a } \\
22 \text { años. } \\
45.6 \% \text { hombres } \\
54.4 \% \text { mujeres }\end{array}$ & 212 & - & - & $8.61 \%$ & - & - & - \\
\hline
\end{tabular}

CIUDAD DE MÉXICO

\begin{tabular}{|c|c|c|c|c|c|c|c|c|c|c|c|}
\hline $\begin{array}{l}\text { Rivera, \& } \\
\text { Andrade. }\end{array}$ & 2006 & - & $\begin{array}{c}263 \\
\text { estudiantes } \\
\text { Muestra No } \\
\text { aleatoria }\end{array}$ & $\begin{array}{c}-\overline{5} \\
30.54 \% \\
\text { hombres. } \\
69.58 \% \\
\text { mujeres }\end{array}$ & 42 & 6 & 36 & - & - & - & - \\
\hline $\begin{array}{l}\text { Palacios, \& } \\
\text { Andrade. }\end{array}$ & 2007 & - & $\begin{array}{c}1,000 \\
\text { estudiantes } \\
\text { Muestra No } \\
\text { aleatoria }\end{array}$ & $\begin{array}{c}\text { De } 14 \text { a } 22 \\
\text { años } 48.5 \% \\
\text { hombres. } \\
51.5 \% \text { mujeres }\end{array}$ & - & - & - & $12.30 \%$ & - & - & - \\
\hline
\end{tabular}

Palacios,

Sánchez, \& $2010 \quad$ - $\quad$ estudiantes

Andrade.

$\begin{array}{llcc}\begin{array}{l}\text { González- } \\ \text { Fuentes, \& }\end{array} & 2013 & - & \begin{array}{c}665 \\ \text { estudiantes } \\ \text { Andrade. }\end{array} \\ & & & \begin{array}{c}\text { Muestra No } \\ \text { aleatoria }\end{array} \\ \begin{array}{l}\text { Monge, } \\ \text { Cubillas, } \\ \text { Román, \& } \\ \text { Valdez. }\end{array} & 2007 & - & \begin{array}{c}1,358 \\ \text { estudiantes } \\ \text { Muestrea } \\ \text { aleatoria }\end{array} \\ & & & \end{array}$

De 14 a 23

años $47.27 \%$

hombres.

$52.72 \%$

mujeres

De 14 a 20

años $50 \%$

hombres. $50 \%$ mujeres

\section{$\begin{array}{lll}79 & 24 & 55\end{array}$}

\section{SONORA}

De 15 a los 19 años de edad $41.9 \%$

hombres

$58.1 \%$ mujeres

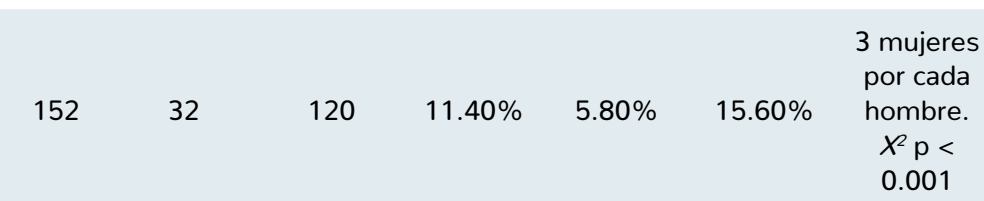

LAS 32 ENTIDADES FEDERATIVAS DE LA REPÚBLICA MEXICANA 
Revisión de la literatura | Intento suicida: Veinticinco años de investigación... | Córdova-Osnaya, Rosales-Pérez \& Rosales-Córdova

\begin{tabular}{|c|c|c|c|c|c|c|c|c|c|c|c|}
\hline \multirow[b]{2}{*}{$\begin{array}{l}\text { AutORES } \\
\text { DEL } \\
\text { ARTículo }\end{array}$} & \multirow{2}{*}{$\begin{array}{l}\text { AÑo } \\
\text { DE } \\
\text { PUBLI- } \\
\text { CACIÓN }\end{array}$} & \multirow{2}{*}{$\begin{array}{l}\text { AÑo DE } \\
\text { CAPTURA } \\
\text { DE LOS } \\
\text { DATOS }\end{array}$} & \multirow{2}{*}{$\begin{array}{c}\text { TAMAÑO DE } \\
\text { LA MUESTRA } \\
\text { Y TIPO DE } \\
\text { MUESTREO }\end{array}$} & \multirow[b]{2}{*}{$\begin{array}{l}\text { EDAD Y \% DE } \\
\text { PARTICIPANTES } \\
\text { POR SEXO }\end{array}$} & \multicolumn{3}{|c|}{$\begin{array}{l}\text { REPORTE DE INTENTO SUICIDA } \\
\text { CUANDO MENOS UNA VEZ } \\
\text { EXPRESADO EN FRECUENCIA }\end{array}$} & \multicolumn{3}{|c|}{$\begin{array}{c}\text { RePORTE de INTENTO SUICIDA } \\
\text { CUANDO MENOS UNA VeZ } \\
\text { EXPRESADO EN PORCENTAJE }\end{array}$} & \multirow{2}{*}{$\begin{array}{c}\text { RAZÓN DE } \\
\text { INTENTO } \\
\text { SUICIDA } \\
\text { MUJER/ } \\
\text { HOMBRE }\end{array}$} \\
\hline & & & & & TOTAL & HOMBRES & Mujeres & TOTAL & $\begin{array}{c}\text { HOMBRES } \\
\text { RESPECTO } \\
\text { AL TOTAL } \\
\text { DE } \\
\text { HOMBRES }\end{array}$ & $\begin{array}{c}\text { MUJERES } \\
\text { RESPECTO } \\
\text { AL TOTAL } \\
\text { DE } \\
\text { MUJERES }\end{array}$ & \\
\hline $\begin{array}{l}\text { Pérez- } \\
\text { Amezcua } \\
\text { et al. }\end{array}$ & 2010 & 2007 & $\begin{array}{l}12,424 \\
\text { estudiantes } \\
\text { Muestra } \\
\text { aleatoria }\end{array}$ & $\begin{array}{c}\text { De } 14 \text { a } 19 \\
\text { años } 45 \% \\
\text { hombres } 55 \% \\
\text { mujeres }\end{array}$ & 1,093 & - & - & $9.00 \%$ & - & - & - \\
\hline \multicolumn{12}{|c|}{ CHIAPAS: CIUDAD DE TUXTLA GUTIERREZ } \\
\hline $\begin{array}{l}\text { Cantoral- } \\
\text { Cancino, \& } \\
\text { Betancourt- } \\
\text { Ocampo. }\end{array}$ & 2011 & - & $\begin{array}{c}\quad 317 \\
\text { estudiantes } \\
\text { Muestra No } \\
\text { aleatoria }\end{array}$ & $\begin{array}{c}\text { De } 14 \text { a } 19 \\
\text { años } 47.9 \% \\
\text { hombres. } \\
52.1 \% \text { mujeres }\end{array}$ & - & - & - & $9.10 \%$ & - & - & - \\
\hline
\end{tabular}

\section{Tabla 2}

Descripción de la información de dos artículos que utilizaron la forma de registrar el intento suicida por medio de dos preguntas: "En los últimos cinco años, ¿has intentado alguna vez quitarte la vida?" aplicada en el estado de Colima, y "En los últimos 12 meses, ¿las veces que ha intentado suicidarse en realidad?" correspondiente a la región centro sur de Jalisco.

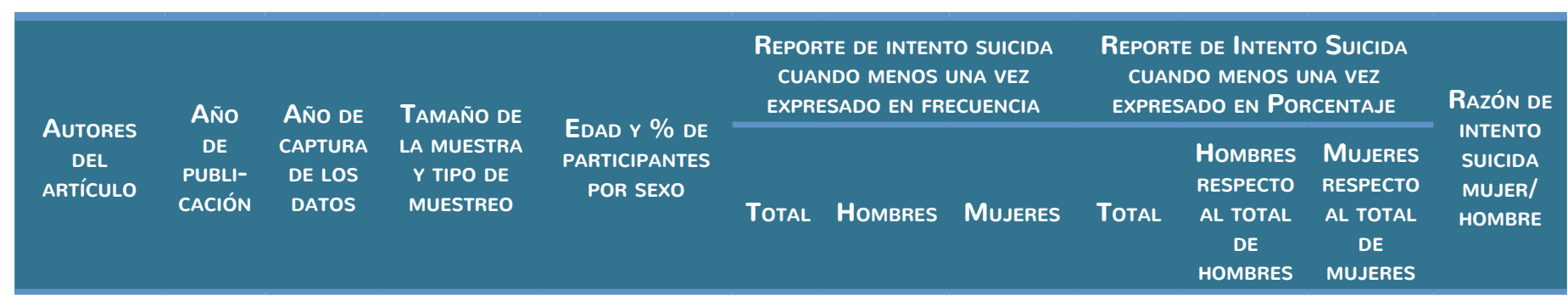

COLIMA

\begin{tabular}{|c|c|c|c|c|c|c|c|c|c|c|c|}
\hline $\begin{array}{l}\text { Espinoza- } \\
\text { Gómez et } \\
\text { al. }\end{array}$ & 2010 & 2006 & $\begin{array}{c}5,484 \\
\text { estudiantes } \\
\text { todos los } \\
\text { de primer } \\
\text { ingreso }\end{array}$ & $\begin{array}{c}\text { De } 14 \text { a } 19 \\
\text { años de edad } \\
46 \% \text { hombres } \\
54 \% \text { mujeres }\end{array}$ & - & 111 & 289 & $7.30 \%$ & - & - & - \\
\hline
\end{tabular}

\section{REGIÓN CENTRO SUR JALISCO: CIUDAD GUZMÁN}

\begin{tabular}{|c|c|c|c|c|c|c|c|c|c|c|c|}
\hline $\begin{array}{l}\text { Hidalgo- } \\
\text { Rasmussen, } \\
\text { \& Hidalgo- } \\
\text { San Martín. }\end{array}$ & 2015 & - & $\begin{array}{c}889 \\
\text { estudiantes } \\
\text { Muestra No } \\
\text { aleatoria }\end{array}$ & $\begin{array}{c}\text { De } 14 \text { a } 18 \\
\text { años } 45 \% \\
\text { hombres } \\
55 \% \text { mujeres }\end{array}$ & - & - & - & $7.60 \%$ & $5.20 \%$ & $9.70 \%$ & $\begin{array}{c}\text { Dos } \\
\text { mujeres } \\
\text { por cada } \\
\text { hombre. } \\
\text { Razón de } \\
\text { momios, } \\
\text { OR }=2 \text {, p } \\
<0.05\end{array}$ \\
\hline
\end{tabular}


Tabla 3

Descripción de la información de los artículos que no especificaron la forma de registrar el intento suicida.

\begin{tabular}{|c|c|c|c|c|c|c|c|c|c|}
\hline $\begin{array}{l}\text { AutORES } \\
\text { DEL } \\
\text { ARTÍ́culo }\end{array}$ & $\begin{array}{l}\text { AÑo } \\
\text { DE } \\
\text { PUBLI- } \\
\text { CACIÓN }\end{array}$ & $\begin{array}{l}\text { AÑO DE } \\
\text { CAPTURA } \\
\text { DE LOS } \\
\text { DATOS }\end{array}$ & $\begin{array}{l}\text { TAMAÑO DE } \\
\text { LA MUESTRA } \\
\text { Y TIPO DE } \\
\text { MUESTREO }\end{array}$ & $\begin{array}{l}\text { EDAD Y \% DE } \\
\text { PARTICIPANTES } \\
\text { POR SEXO }\end{array}$ & $\begin{array}{l}\text { REPORTE DE INTENTO SUICIDA } \\
\text { CUANDO MENOS UNA VEZ } \\
\text { EXPRESADO EN FRECUENCIA } \\
\text { TOTAL HOMBRES MUJERES }\end{array}$ & \multicolumn{3}{|c|}{$\begin{array}{c}\text { Reporte de Intento SUICIDA } \\
\text { CUANDO MENOS UNA VEZ } \\
\text { EXPRESADO EN PORCENTAJE }\end{array}$} & $\begin{array}{c}\text { RAZÓN DE } \\
\text { INTENTO } \\
\text { SUICIDA } \\
\text { MUJER/ } \\
\text { HOMBRE }\end{array}$ \\
\hline
\end{tabular}

JALISCO: Ciudad de Guadalajara

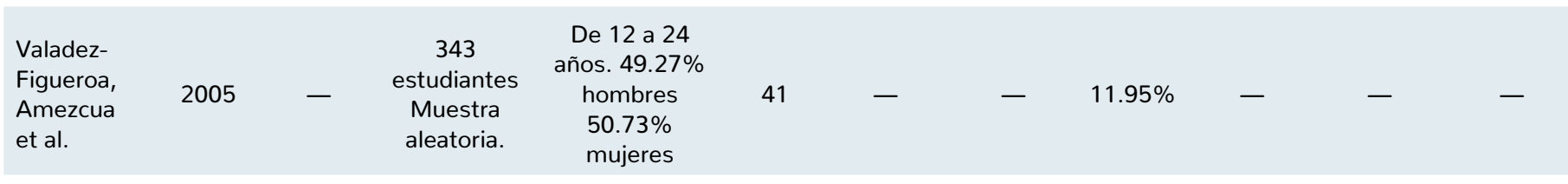

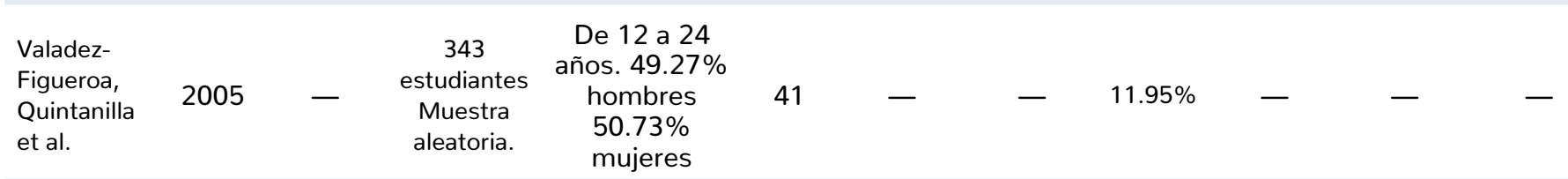

\begin{tabular}{|c|c|c|c|c|c|c|c|c|c|c|}
\hline $\begin{array}{l}\text { Valadez- } \\
\text { Figueroa } \\
\text { et al. }\end{array}$ & 2009 & $\begin{array}{c}723 \\
\text { estudiantes } \\
\text { Muestra } \\
\text { aleatoria }\end{array}$ & $\begin{array}{c}\text { De } 12 \text { a } 24 \\
\text { años. } 46.70 \% \\
\text { hombres } \\
53.30 \% \\
\text { mujeres }\end{array}$ & 72 & 26 & 46 & $9.96 \%$ & $7.70 \%$ & $11.90 \%$ & $\begin{array}{c}1.5 \\
\text { mujeres } \\
\text { por cada } \\
\text { hombre }\end{array}$ \\
\hline
\end{tabular}

\begin{tabular}{|c|c|c|c|c|c|c|c|c|c|c|c|}
\hline $\begin{array}{l}\text { Valadez } \\
\text { et al. }\end{array}$ & 2011 & - & $\begin{array}{c}723 \\
\text { estudiantes } \\
\text { Muestra } \\
\text { aleatoria }\end{array}$ & $\begin{array}{c}\text { De } 12 \text { a } \\
24 \text { años. } \\
46.70 \% \\
\text { hombres } \\
53.30 \% \\
\text { mujeres }\end{array}$ & 79 & - & - & $9.96 \%$ & - & - & - \\
\hline $\begin{array}{l}\text { Valadez- } \\
\text { Figueroa } \\
\text { et al. }\end{array}$ & 2015 & - & $\begin{array}{c}723 \\
\text { estudiantes } \\
\text { Muestra } \\
\text { aleatoria }\end{array}$ & $\begin{array}{l}-46.70 \% \\
\text { hombres } \\
53.30 \% \\
\text { mujeres }\end{array}$ & 72 & - & - & $9.96 \%$ & - & - & - \\
\hline $\begin{array}{l}\text { Valadez- } \\
\text { Figueroa } \\
\text { et al. }\end{array}$ & 2016 & - & $\begin{array}{c}723 \\
\text { estudiantes } \\
\text { Muestra } \\
\text { aleatoria }\end{array}$ & - & 72 & - & - & $9.96 \%$ & - & - & - \\
\hline
\end{tabular}


Revisión de la literatura | Intento suicida: Veinticinco años de investigación... | Córdova-Osnaya, Rosales-Pérez \& Rosales-Córdova

\section{Meta-Análisis del Artículo}




\section{Dimensión Cuantitativa}

\section{Perfil de Evaluación entre pares}
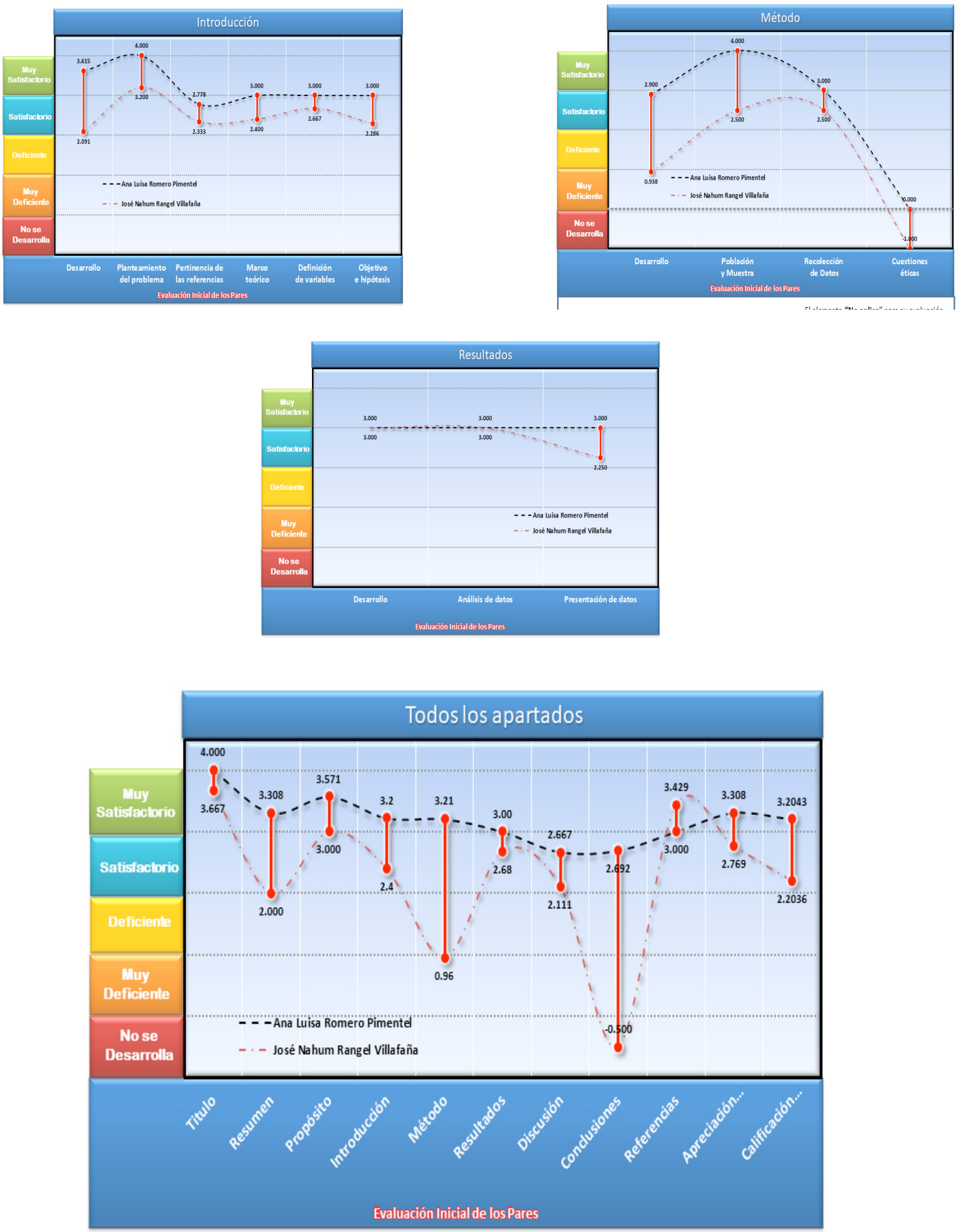

Evaluación Inicialde los Pares 
Revisión de la literatura | Intento suicida: Veinticinco años de investigación... | Córdova-Osnaya, Rosales-Pérez \&Z Rosales-Córdova

\section{Índice de Concordancia}

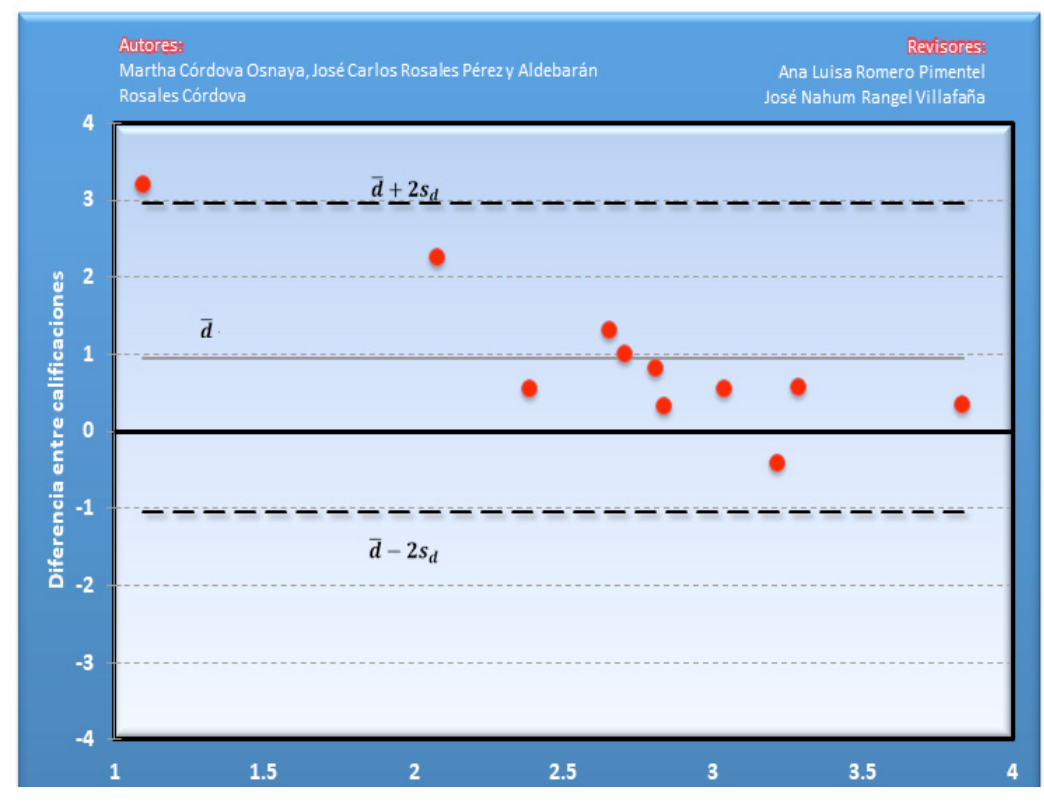

Índice de Acuerdo

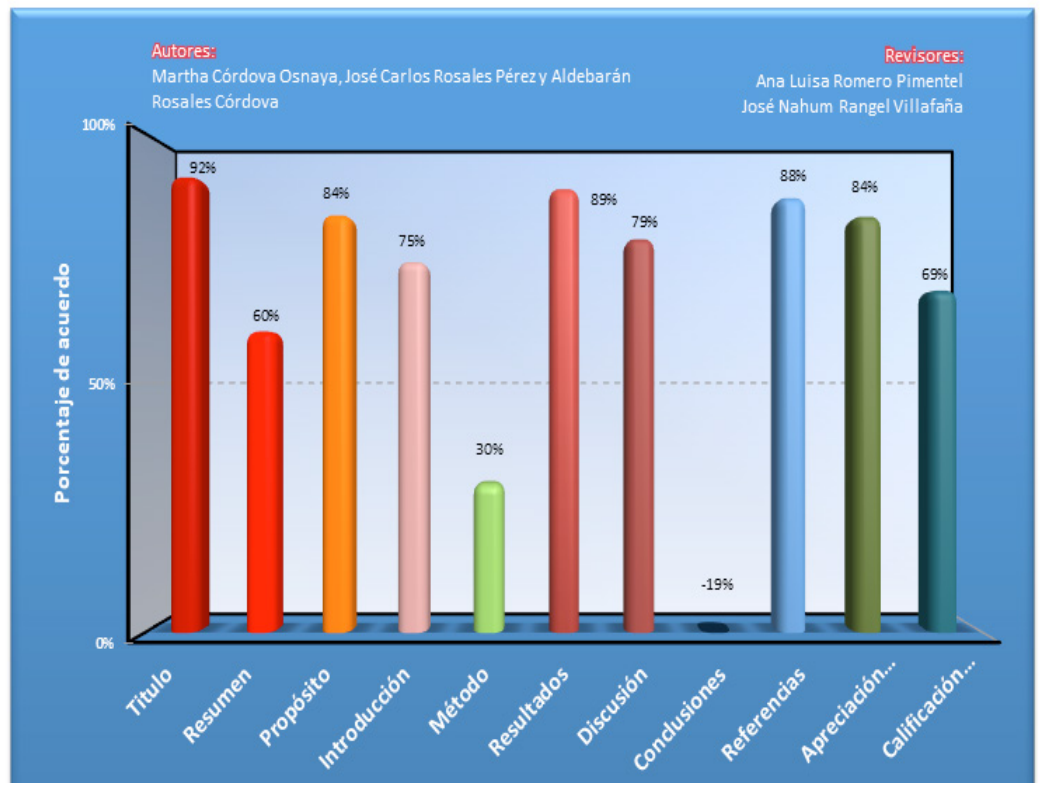


Dimensión Cualitativa

\begin{tabular}{|c|c|}
\hline Revisor 1 & Revisor 2 \\
\hline Ana Luisa Romero Pimentel & José Nahum Rangel Villafaña \\
\hline \multicolumn{2}{|c|}{ Título/Autoría } \\
\hline El titulo es adecuado para el artículo & Sin comentarios \\
\hline \multicolumn{2}{|c|}{ Resumen } \\
\hline $\begin{array}{l}\text { El resumen es adecuado, sin embargo sobre pasa el li- } \\
\text { mite permitido de palabras. }\end{array}$ & $\begin{array}{l}\text { Ambas versiones del resumen sobrepasan el límite de pa- } \\
\text { labras permitidas. - Sugiero precisar el objetivo, pues no } \\
\text { queda claro si la esencia del estudio es describir la forma } \\
\text { en que se ha hecho la investigación o se trata de un análisis } \\
\text { de los resultados obtenidos en dicha investigación. - "Me- } \\
\text { dir el intento de suicidio", se entiende que se busca medir } \\
\text { la frecuencia de la conducta, pero parece que los resulta- } \\
\text { dos son poco homogéneos. Sugiero usar la frase se regis- } \\
\text { tró. - Es pertinente la mencionar la conclusión del estudio. }\end{array}$ \\
\hline \multicolumn{2}{|c|}{ Próposito del Estudio } \\
\hline $\begin{array}{l}\text { El propósito del estudio es claro y adecuado para el ar- } \\
\text { tículo }\end{array}$ & $\begin{array}{l}\text { Pienso que el propósito del estudio es demasiado amplio y } \\
\text { por ende difícil de abordar en su totalidad. El escrito tien- } \\
\text { de a ser pesado y confuso. La atención del lector se pierde } \\
\text { en el mar de información. Sugiero afinar el objetivo del } \\
\text { estudio para reportar resultados y discusión más precisa y } \\
\text { destacando la información más relevante para el objetivo. }\end{array}$ \\
\hline \multicolumn{2}{|c|}{ Introducción } \\
\hline $\begin{array}{l}\text { La introducción es adecuada, sin embargo, carece de la } \\
\text { información de aquellos estudios similares a este rea- } \\
\text { lizados en México, es importante contrastar dicha in- } \\
\text { formación, por ejemplo; Vázquez-Vega, D., Piña-Pozas, } \\
\text { M., González-Forteza, C., Jiménez-Tapia, A. \& Mondra- } \\
\text { gón-Barrios, L. (2015). La investigación sobre } 2 / 8 / 2019 \\
\text { Instrucciones de Dictaminación de un Manuscrito } \\
\text { Enviado a la RDIPyCS cuved.unam.mx/campus/mod/ } \\
\text { questionnaire/print.php? qid=423\&rid=89322\&cour- } \\
\text { seid=316\&sec=1 10/2116 suicidio en México en el pe- } \\
\text { riodo 1980-2014: análisis y perspectivas. Acta Univer- } \\
\text { sitaria, 25(NE-2), 62-69. doi: } 10.15174 / \text { au.2015.855 }\end{array}$ & $\begin{array}{l}\text { Parte de la temática abordada en los primeros párrafos, } \\
\text { se resuelve en la literatura relacionada con el Sistema de } \\
\text { Clasificación de Violencia Autodirigida y la literatura an- } \\
\text { tecedente a este de Silverman y O 'Carrol. - Según las re- } \\
\text { ferencias anteriores y algunas más (Nock), el concepto de } \\
\text { suicidio consumado esta actualmente de desuso. En reali- } \\
\text { dad se sugiere no utilizarlo, pues se trata de un pleonasmo. } \\
\text {-Me parece que uno de los aportes más relevantes del es- } \\
\text { tudio es evidenciar los errores y dificultad para operacio- } \\
\text { nalizar y registrar el intento de suicidio. Sin embargo, esta } \\
\text { idea se diluye entre toda la información que se presenta. }\end{array}$ \\
\hline
\end{tabular}


Revisión de la literatura | Intento suicida: Veinticinco años de investigación... | Córdova-Osnaya, Rosales-Pérez \&Z Rosales-Córdova

\begin{tabular}{|c|c|}
\hline Revisor 1 & Revisor 2 \\
\hline \multicolumn{2}{|c|}{ Método } \\
\hline $\begin{array}{l}\text { Sería más claro si se anexa un rubro de análisis estadistico } \\
\text { donde se describa cómo analizaron los datos. }\end{array}$ & $\begin{array}{l}\text { El método pudiera ser más claro y fácil de replicar si se } \\
\text { siguen los lineamientos de la universidad de Oxford para } \\
\text { hacer una revisión sistemática. Tal vez no logra serlo pero } \\
\text { seguir dichos lineamientos ayudará a la presentación del } \\
\text { trabajo. }\end{array}$ \\
\hline \multicolumn{2}{|c|}{ Resultados } \\
\hline $\begin{array}{l}\text { Como comenté anteriormente, sería pertinente agregar } \\
\text { un rubro de análisis estadístico, donde se justifique el } \\
\text { como se analizaron los datos. }\end{array}$ & $\begin{array}{l}\text { Las tablas mostradas tienden a ser repetitivas y no parecen } \\
\text { indispensables }\end{array}$ \\
\hline \multicolumn{2}{|c|}{ Discusión } \\
\hline $\begin{array}{l}\text { La discusión puede ser enriquecida si se hace una com- } \\
\text { paración con estudios internacionales. Además, un } \\
\text { gran aporte a la comunidad cientifica sería plantear ba- } \\
\text { sados en la literatura un flujograma de algún propuesta } \\
\text { teórica de cuales elementos ustedes consideran deben } \\
\text { emplearse para la estandarización de futuros estudios } \\
\text { de conducta suicida en adolescentes mexicanos. }\end{array}$ & $\begin{array}{l}\text { AL reportar tantos resultados, la discusión también se tor- } \\
\text { na confusa y no se evidencia con claridad la relevancia de } \\
\text { los hallazgos y sus efectos. }\end{array}$ \\
\hline \multicolumn{2}{|c|}{ Conclusiones } \\
\hline $\begin{array}{l}\text { Sería interesante agregar las aportaciones del estudio a } \\
\text { la comunidad cientifica }\end{array}$ & $\begin{array}{l}\text { Se propusieron líneas de investigación nuevas pero no en } \\
\text { este apartado. Parece que en las conclusiones solo se repi- } \\
\text { ten de forma resumida los resultados. }\end{array}$ \\
\hline \multicolumn{2}{|c|}{ Referencias } \\
\hline Las referencias son adecuadas & Ninguno de los artículos electrónicos cuentan con doi. \\
\hline
\end{tabular}




\section{EDITORIAL}

